\title{
¿EDUCACIÓN INCLUSIVA O INCLUSIÓN EDUCATIVA? REFLEXIONES ACERCA DE LA EDUCACIÓN INDÍGENA EN MÉXICO
}

ENRIQUE BAUTISTA ROJAS ${ }^{1}$

\section{RESUMEN}

En México, las escuelas indígenas han sido históricamente escenarios con diversas dificultades y retos para poder garantizar la educación de niñas, niños y jóvenes de las comunidades indígenas. En consideración de ello, el trabajo explora la situación de la educación indígena para analizar la forma en que ésta se ha traducido en acciones de desatención y aculturación. Los referentes parten de la revisión y análisis de literatura especializada y se retoman planteamientos de la Educación intercultural y la Educación inclusiva en relación con las poblaciones indígenas. Se busca motivar la reflexión para mirar a las escuelas indígenas desde una perspectiva contextualizada donde la diversidad represente oportunidades para la formación de niñas, niños y jóvenes de manera integral.

Palabras clave: comunidades indígenas, educación indígena, educación inclusiva, interculturalidad.

\footnotetext{
1 Licenciado en Pedagogía. Facultad de Estudios Superiores Acatlán de la Universidad Nacional Autónoma de México (México). Avenida Paseo de la Reforma 122, Colonia Juárez, Delegación Cuauhtémoc. Código Postal: 06600 Ciudad de México, México. Correo electrónico: kique_pedagogo.unam@hotmail.com
} 


\title{
INCLUSIVE EDUCATION OR EDUCATIONAL INCLUSION? REFLECTION ABOUT THE INDIGENOUS EDUCATION IN MÉXICO
}

\section{ABSTRACT}

In Mexico, indigenous schools have historically been scenarios with various difficulties and challenges to ensure that the education of children and young people of indigenous communities. In consideration of this, the research explores the situation of indigenous education to analyze the way in which this has resulted in neglect and acculturation activities. The referents based on the review and analysis of specialized literature and the approaches taken from intercultural education and inclusive education in relation to indigenous communities. It aims to motivate the reflection to look at the indigenous schools from a perspective contextualized where diversity represents opportunities for the training of children and young people in a holistic manner.

Keywords: indigenous communities, indigenous education, inclusive education, intercultural.

\section{Introducción}

\begin{abstract}
Nuestra realidad es amarga y triste, la escuela ha matado en las mentes y corazón de las generaciones jóvenes la realidad y el orgullo de que somos indios; han obligado a que nuestros hijos olviden, inclusive, desprecien nuestros idiomas, nuestras formas de vestir, nuestra lucha diaria de sobrevivencia las costumbres de la comida, bailes antiguos, las medicinas de nuestros antepasados y hasta nuestro color y rasgos físicos; han hecho a un lado tequios, sistemas de cargo, ceremonias religiosas antiguas, historias propias (Pérez et. al., 2009, pp.
\end{abstract} 182-183)

México representa un mosaico compuesto por una multiplicidad de pueblos indígenas con diversas características culturales, los cuales desde hace siglos y hasta ahora, forman parte de la multicultural del país, así reconocida desde la Carta Magna. Actualmente cerca de 15 millones de 
personas de 3 años y más (Inegi, 2011) pertenecen a un grupo indígena y existen 68 lenguas indígenas que se derivan en 364 variantes lingüísticas, algunas de las cuales se encuentran en situación de desaparición. Desde 1992, el país fue reconocido como una nación pluricultural y tras una reforma constitucional, se reconocieron los derechos de las comunidades indígenas. De acuerdo con la Constitución Política de los Estados Unidos Mexicanos:

La Nación tiene una composición pluricultural sustentada originalmente en sus pueblos indígenas que son aquellos que descienden de poblaciones que habitaban en el territorio actual del país al iniciarse la colonización y que conservan sus propias instituciones sociales, económicas, culturales y políticas, o parte de ellas... Esta Constitución reconoce y garantiza el derecho de los pueblos y las comunidades indígenas a la libre determinación (artículo $2^{\circ}$ ).

A pesar de este reconocimiento, el problema de la discriminación y violencia hacia los grupos indígenas es una realidad que impera en el país. Los cambios no han sido posibles como pudiera esperarse no obstante de lo establecido legalmente; las ideas y concepciones discriminatorias tienen una larga historia en el país y su arraigo entre las personas continúa vigente. Al respecto, señalan Tovar y Avilés (2005) que:

Pese a que México jurídicamente se reconoce como una nación pluricultural, [...] el reconocimiento de la multiculturalidad no ha tenido efectos significativos que modifiquen el hecho de que entre la cultura de la sociedad mayoritaria y las de los pueblos indígenas se generen relaciones de carácter discriminatorio y segregacionista y se perciba la diversidad como problema a superar. (p. 249)

Históricamente, las comunidades indígenas representan un sector de la población que ha vivido diferentes problemáticas desde el despojo territorial y material a la llegada europea, hasta situaciones como la marginación, la pobreza, la discriminación, el analfabetismo, la falta de acceso a servicios de salud y educación. En este sentido, el Consejo Nacional de Evaluación de la Política de Desarrollo Social - Coneval (2012) indica que:

Resulta inevitable resaltar el hecho de que, así sean siete, once o dieciocho millones de personas, se trata de individuos que viven en condiciones de rezago que se traducen en la vulneración sistemática de derechos sociales fundamentales. Resulta preocupante que la sistematicidad de estas violaciones parece asociarse con pertenecer a un grupo culturalmente distinto que acumula desventajas sociales. (pp. 144-145) 
Por otro lado, el problema de la discriminación sigue siendo un obstáculo importante para la inclusión, reconocimiento y valoración de las comunidades indígenas para posibilitar un desarrollo más armónico entre las personas. En ocasiones, las concepciones discriminatorias pasan a formar parte de las políticas públicas (como la educativa), tanto de forma sutil como evidente, generando así mecanismos institucionales que sostienen dichos prejuicios. De este modo, en distintos espacios laborales y educativos, entre otros, se coartan el acceso y la participación en igualdad de condiciones de las personas con origen étnico. De acuerdo con Stavenhagen (2001):

En el ámbito institucional suele mencionarse el funcionamiento de instituciones públicas y privadas en que reciben trato diferenciado los miembros de determinadas minorías y grupos sociales definidos con criterios étnicos y raciales. Por ejemplo, el caso de las instituciones de educación superior, algunos tipos de empleos, la vivienda exclusiva, a los que no se permite la entrada de personas pertenecientes a grupos identificados como "distintos" o "inferiores" a la norma hegemónica. (p. 4)

Según señalan los datos de la Encuesta Nacional sobre Discriminación en México (Enadis) 2010, elaborada por el Consejo Nacional para Prevenir la Discriminación (Conapred), el $23.4 \%$ de la población no estaría dispuesta a permitir que en su casa vivieran personas con una cultura distinta. En cuanto a la percepción que tienen las personas pertenecientes a comunidades indígenas, la misma encuesta (Conapred, 2010, p. 113) evidencia que la discriminación ${ }^{2}$ es el principal problema que tienen (Gráfica 1). En cuanto a educación, uno de cada cuatro dijo no tener las mismas oportunidades para tener acceso a servicios de salud o educación.

\footnotetext{
${ }^{2}$ De acuerdo con la "Ley federal para prevenir y eliminar la discriminación" de México, la discriminación incluye la distinción, exclusión, restricción o preferencia, ya sea por acción u omisión. Entre los motivos de ella, se encuentran el origen étnico o nacional, el color de piel, la cultural, el sexo, el género, la edad, la condición social, discapacidades, la lengua, entre otros (Conapred, 2014, pp. 19-20).
} 
Figura 1. Principales problemas que reportan los integrantes comunidades indígenas

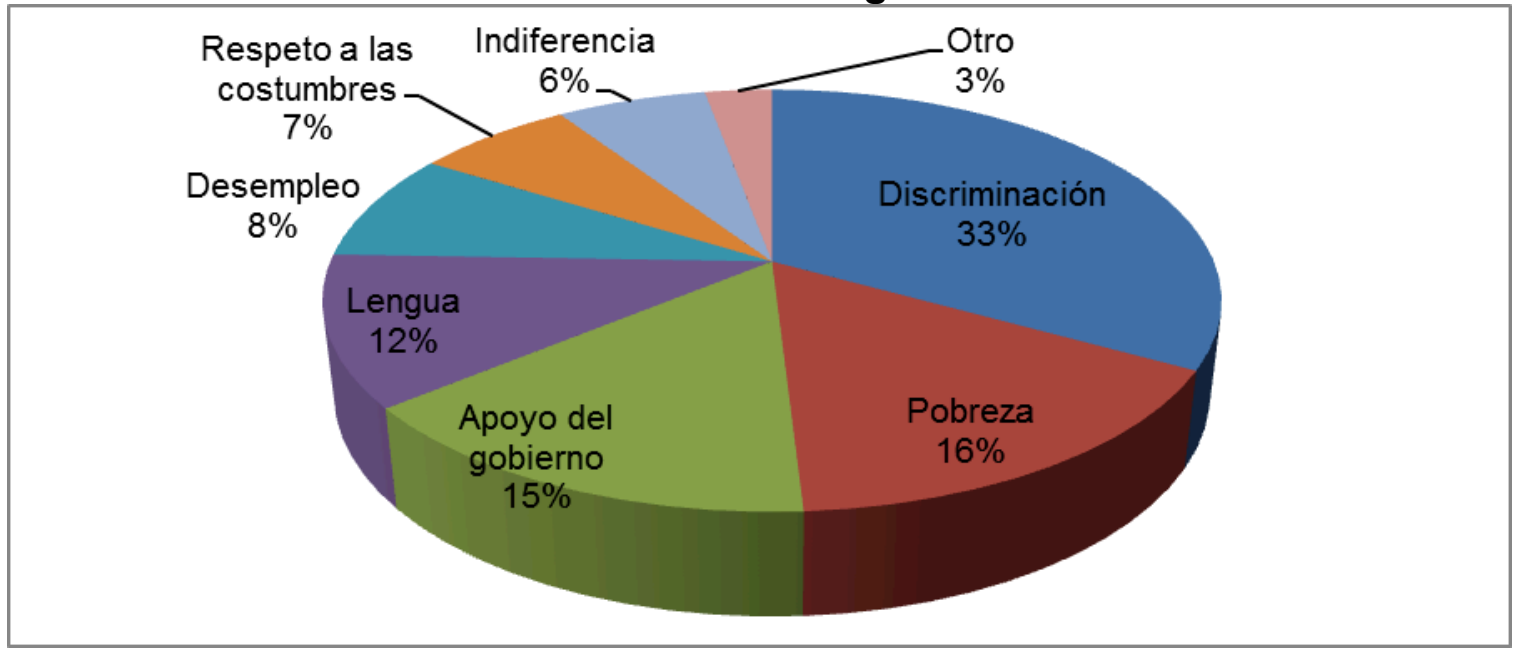

Fuente: Elaborada con base en Conapred (2010, p. 113).

En consideración de lo expuesto, el documento explora la situación de la educación indígena en México para analizar la forma en que ésta se ha traducido en acciones que han incrementado la cobertura escolar en las comunidades indígenas, pero con tendencias hacia la homogeneización a partir de planteamientos etnocéntricos. Se deja de lado la práctica de acciones desde una perspectiva contextualizada y que reconozca los derechos de las niñas, niños y jóvenes indígenas. A la vez, se enfatiza en la forma en cómo las condiciones de las escuelas indígenas, los materiales y la organización escolar representan retos a atender para un funcionamiento más adecuado de estas escuelas, pues el desarrollo pleno de los conocimientos, habilidades, valores y actitudes de los estudiantes se ven dificultados ante situaciones de inequidad y desigualdad.

Se discurre acerca de la necesidad de mirar a las escuelas indígenas a partir de planteamientos derivados de la educación inclusiva y la educación intercultural, donde la diversidad represente oportunidades para la formación de niñas, niños y jóvenes de manera integral. En este sentido, se entiende por Educación intercultural la propuesta alterna a los procesos de la educación homogeneizante y que busca recuperar los elementos culturales de las comunidades indígenas. Ello implica, la consideración del contexto social y cultural de los estudiantes como elementos relevante en los procesos 
educativos; asimismo, avanza más allá del mero reconocimiento de los elementos culturales para problematizar y llevar al aula los aspectos sociales, políticos y económicos que han afectado a los grupos originarios históricamente con la intención política de generar conciencia y posibilidades de transformación social.

Por su parte, pero en sentido conjunto, la educación inclusiva busca garantizar el acceso y permanencia, pero además, la participación de todas las personas en el sistema educativo, con especial énfasis en las poblaciones marginadas o excluidas, en un contexto de respeto y valoración de la diversidad. Busca garantizar el derecho a la educación de los grupos en situación de vulnerabilidad (las personas en sí no son vulnerables, sino son las condiciones que les rodean lo que generan dicha situación). Parte del supuesto de que las diversas condiciones sociales, políticas y económicas representan barreras que limitan el aprendizaje, por lo que la atención deberá asegurar la eliminación de las mismas generando condiciones de equidad y justicia social.

El trabajo consiste en una reflexión teórica, sustentada en la revisión y análisis de la literatura especializada en el tema, así como en la recuperación de la experiencia obtenida a partir de observaciones informales en escuelas y comunidades indígenas. Para el análisis se retoman planteamientos derivados de la interculturalidad y la educación inclusiva en relación a las poblaciones indígenas. Se presentan en un primer momento algunas consideraciones en relación a los planteamientos en relación a la educación indígena en México. Posteriormente se brinda un panorama de la situación de las escuelas que atienden a estas comunidades, destacando los problemas y desafíos que tienen. Por último, se analizan y discuten algunos aspectos de la educación indígena a partir de los planteamientos de la interculturalidad y la inclusividad a fin de motivar la reflexionar sobre la necesidad de transformación para la generación de un proyecto educativo más acorde a la diversidad, que la respete y valore. 


\section{Incluir a los indígenas: La educación homogeneizante}

En diferentes momentos de la historia en México, pocas fueron las acciones educativas destinadas a las poblaciones indígenas; y las existentes, tenían como propósito la incorporación de la población indígena al "proyecto de nación”, a partir de su asimilación mediante la eliminación de sus aspectos culturales, considerados pocos acordes con las formas sociales y culturales. Sin embargo, la integración buscaba a la vez mantener a los indígenas en los espacios rurales, asignando así un "rol" que debía ser cumplido dentro de las actividades que llevaran al progreso nacional.

Desde la llegada europea y a lo largo del periodo virreinal, la educación de las poblaciones indígenas estuvo destinada a la aculturación y al exterminio de las prácticas y creencias consideradas no adecuadas por los conquistadores, principalmente en lo referente al idioma y la religión. Este aspecto poco a poco se fue descuidando y en años previos al inicio del movimiento de Independencia (1810), muchas poblaciones indígenas y rurales habían sido abandonadas, por lo que existía una alta tasa de población que no hablaba español (Antolínez, 2013).

Hacia los años de la independencia de México (1821), las poblaciones pequeñas e indígenas estaban abandonadas y no existía preocupación mayor al respecto de ellas. A finales del siglo XIX y principios del $\mathrm{XX}$, mediante programas que buscaban la integración nacionalista a través de la educación, se emprendieron acciones para enseñar el español a los indígenas como forma de construir una ciudadanía conforme al proyecto nacional de aquel entonces.

Al llegar el siglo $X X$ y terminado el movimiento revolucionario iniciado en 1910, se buscó integrar a los indígenas a través de diferentes acciones educativas, principalmente encaminadas a la castellanización, pues ante las diversas carencias que tenían estas poblaciones y la creencia de que tenían menores posibilidades para aprender, bastaba con una alfabetización inicial en castellano.

Para Gamio (1883-1860), era necesario tener una visión más realista respecto a la educación, reemplazando la idea de que el alfabetismo en las zonas indígenas y rurales traería consigo de manera casi milagrosa del 
progreso; asimismo, se mostraba en contra de la idea prejuiciosa de que era causa del atraso nacional la presencia de los indígenas. Su posicionamiento buscaba la homogeneización de la cultura a partir de la integración, o mestizaje, de las comunidades indígenas. Según este autor:

Se predica con frecuencia que de la «alfabetización» de todos los mexicanos dependen el bienestar nacional y el engrandecimiento de la patria. Desde luego, no aceptamos que el factor educativo produzca tales milagros, si no está acompañado de factores complementarios como el político, el económico, el étnico y otros [...]

Para que la evolución cultural de un pueblo sea normal, es indispensable que todos los elementos que constituyen a la población se eduquen a la vez, y esto sólo se consigue implantando la educación integral [...] ¿Debe implantarse o no la educación integral en México? Sí, debe implantarse, pero con un previo y sólido conocimiento de la población en la cual se va a implantar. (Gamio, 1916, pp. 285-286)

Otro de los pensadores que se preocupó por la educación indígena y rural en aquellos años fue Moisés Sáenz (1888-1941), quien además introdujo al país algunas ideas provenientes de teóricos norteamericanos respecto a la educación experimental (desarrollado por John Dewey). Saénz creía que una forma de cerrar las brechas sociales era atender a las escuelas rurales como forma de integrar a la población, sobre todo indígena, bajo afirmaciones como "civilizar" y "uniformar". Sáenz (1939) señalaba:

Pretendo sencillamente que el indio sea considerado como un dato, como un factor real o importante del problema de la integración de México. Soy partidario ferviente de la 'incorporación' del indio a la familia mexicana, si esto quiere decir, en lo biológico, el proceso natural del mestizaje; en lo político, dar al indio cabida libre, con un criterio igualitario y democrático, al campo de la ciudadanía, y en lo cultural, una amalgama consciencia y respetuosa, a la vez que selectiva e inteligente, de los rasgos y valores autóctonos con los elementos típicos y normativos del diseño cultural mexicano. (p. 213)

De manera específica, para el caso de las escuelas rurales que carecían de maestros para cada grado, Rafael Ramírez (1885-1859) promovió diversas medidas y métodos para favorecer la enseñanza en esas escuelas, como "que cada maestro estableciera su propio programa, que se centraran en proyectos vinculados a la comunidad y utilizaran «métodos naturales» para la enseñanza 
de la lengua y la aritmética, y proyectos comunitarios para formar a los niños «para la vida»" (Rockwell y Garay, 2007). En algunos casos, los maestros de escuelas rurales lograron llevar a cabo proyectos comunitarios; no obstante, por mucho tiempo continuo permeando la visión y las propuestas para escuelas urbanas y de organización completa.

En estos mismos años, diversos proyectos tuvieron lugar en el país como el establecimiento de la primera Casa del Estudiante Indígena en 1925, la creación del Departamento de Educación y Cultura Indígena en 1934 o el surgimiento del Proyecto Tarasco en 1939 que logró algunos resultados en la castellanización a través de la alfabetización en lenguas indígenas (Martínez, 2011, p. 2). Sin embargo, este último fue abandonado por muchos años e incluso se llegó a prohibir que los niños hablaran en su lengua materna en las escuelas.

Si bien durante el periodo cardenista (1934-1940) se "reconoció al indio como ser social capaz de integrarse a la nación sin menoscabo de su cultura" (Martínez, 2011, p. 2), los posteriores periodos se mostraron en contra del bilingüismo y el reconocimiento de la diversidad cultural, sobre todo con la llegada del discurso y las acciones para la Modernización nacional. De acuerdo con Gutiérrez (2006):

La misión de la escuela formal debía ser des-indianizar al Indio, en aras de la "modernización" y "desarrollo nacional", y promover - a través de las metas contenidas en los programas educativos correspondientes - la progresiva sedentarización y "urbanización" de las comunidades rurales indígenas, el "mestizaje" sociocultural de sus miembros, y su incorporación - como "mano de obra" asalariada - en la economía capitalista imperante. (p.2)

Hacia 1963 se recuperó la política bilingüe y las técnicas y métodos del Proyecto; no obstante, diversas problemáticas impidieron su correcta implementación. En 1978 la SEP creó la Dirección General de Educación Indígena (DGEI), como responsable de los proyectos educativos, capacitación, metodologías, técnicas y programas para las niñas y niños indígenas. Hacia principios de los años ochenta, se definieron bases generales para la educación indígena que dieron lugar a un modelo bilingüe bicultural; éste consistía en la enseñanza de dos lenguas y dos culturas de forma simultánea, 
"pero sin metodología específica, ni estrategias didácticas, ni materiales, ni mucho menos con un personal profesionalizado con la formación y capacidad suficiente para enfrentar dicho reto en el nivel nacional" (Villagómez y Nuño, 2009, p. 210).

En la Ley General de Educación de 1993 se reconoció la importancia de las lenguas indígenas en el país, sin embargo se carecía de un programa que respondiera a tal planteamiento. A principios del siglo $X X I$, se instituyó la Educación Intercultural Bilingüe (EIB) y la Ley General de Derechos Lingüísticos de los Pueblos Indígenas que reconocía problemas como la desigualdad social y la importancia de generar cambios para atender problemáticas en comunidades indígenas. Asimismo, reconocían su derecho a ser educados en su lengua, y la importancia de promover el respeto a la pluralidad lingüística de la Nación. Viveros-Márquez y Moreno-Olivos (2014) indican que:

La Educación Intercultural Bilingüe parte de la cultura local del alumno, es decir, de su forma de pensar y de vivir, de su forma de relacionarse con el entorno, de sus costumbres y tradiciones, y del conjunto de símbolos y significados culturales propios. (p.60)

Como se observa, recientemente se han fortalecido las acciones de diversas instituciones relacionadas con la educación indígena y la promoción de las culturas originarias, y actualmente se ha agregado al currículo vigente la asignatura de "Lengua indígena", como espacio para la recuperación de las lenguas y el estudio de las mismas en las aulas indígenas.

Sin embargo, la puesta en marcha de los diferentes acciones ha tenido trabas que han imposibilitado su concreción, pues lo escrito no ha logrado traducirse en acciones que involucren un proyecto pedagógico acorde y congruente. A la vez, de manera velada los proyectos educativos enfocados a los indígenas han continuado con la línea asimilacionista, generando tensiones y discursos contradictorios, pues por un lado se busca impulsar el reconocimiento y valoración, pero por otro homogeneizar según los estándares y principios occidentales enmarcados tanto de manera explícita como "oculta" dentro del currículo. Para Villagómez y Nuño (2009) existe una tendencia a imponer un solo modelo educativo con ciertos criterios etnocéntricos y que 
continúan favoreciendo la castellanización. Desde este posicionamiento, la educación "se convierte en la punta de lanza de la destrucción y desintegración de los grupos indígenas y en la representante de una pseudointegración nacional" (Portal y Valenzuela, 1993, p. 138).

De acuerdo con Tovar y Avilés (2005) "ha predominado una concepción educativa asimilacionista, que se opone a los intentos de aplicar políticas públicas basadas en el pluralismo cultural" (p. 249), por lo que la educación indígena se concibe como un proyecto que desarrollan personas que no pertenecen a comunidades indígenas ni tienen conocimientos o sensibilidad al respecto, generándose así la transmisión de valores, concepciones, creencias, ideas, patrones de conducta, entre otros, desde un contexto y una óptica distinta a la de los grupos originarios. Así, por ejemplo, valores como la colectividad, la comunalidad y la solidaridad, se contraponen con visiones individuales, competitivas y donde el progreso se vincula con la capacidad de movilidad socio-económica.

Se aprecia que "el mundo indígena figura prácticamente relegado al glorioso pasado prehispánico" (Dosil y Lepe, 2011, pp. 4-5), manteniendo de esta forma la admiración al indígena muerto, no al vivo. Ante la función homogeneizante del currículo y su intención de formar individuos estandarizados que respondan a los mismos patrones de conducta, con los mismos conocimientos e, incluso, la misma forma de pensar (Santos, 2009, pp. 191, citado en Jiménez, 2012, p. 11), se deja de lado la heterogeneidad que forma parte de nuestro país. Se preservan los problemas de los alumnos pertenecientes a las minorías, a las cuales generalmente se destina una educación compensatoria, es decir, acciones focalizadas que buscan brindar los mínimos indispensables, pero que en ocasiones "son paliativos reservados sólo a los excluidos" (Hecht, 2007, p. 73).

De este modo, se pretende a través de las políticas educativas que los indígenas aborden los mismos contenidos - en México el currículo tiene un carácter nacional - que aquellos que habitan en otras regiones, sin consideración de las particularidades, necesidades o intereses que tienen estas 
comunidades. En esta línea, el Instituto Nacional de Lenguas Indígenas -Inali (2008) plantea que:

Actualmente los planes de estudio de educación básica son los mismos a nivel nacional, con escasas variantes para adaptarlos a las necesidades específicas de la población a la que va dirigido; asimismo, persiste el criterio homogeneizador que no contempla el respeto a la diversidad, a la pluralidad cultural y al multilingüismo. Vinculado con lo anterior, se observa un desarrollo insuficiente de materiales didácticos en lenguas indígenas con enfoque intercultural y multilingüe. (p. 39)

En este mismo sentido, se puede afirmar que el sistema formal se ve caracterizado por metodologías y contenidos que han sido seleccionados como los únicos valiosos y aceptables para que sean "conocidos" por todos, y genera mecanismos, como exámenes estandarizados, que buscan comprobar que se haya aprendido aquello que desde el centro ha sido definido como válido.

Esto ha traído, entre otros problemas, que las comunidades indígenas tengan los resultados más bajos a nivel nacional dentro de las pruebas que se aplican a nivel en todas las escuelas. Pruebas como Excale (Exámenes de la Calidad y el Logro Educativo), revelan que las menores puntuaciones se encuentran entre los estudiantes que asisten a escuelas indígenas (Galeana, 2013, p. 96). Esto se vincula directamente con actos de discriminación, como lo determinó Conapred en 2011 con la prueba Enlace, al considerar que se cometen actos de este tipo al no tomar en cuenta las necesidades, características propias y la diversidad cultural de los pueblos y comunidades indígenas del país (Hernández, 2013). En su resolución ${ }^{3}$, el Consejo determinó que:

El efecto de los exámenes estandarizados fue que sí generó un tratamiento inequitativo al ser aplicada a niñas y niños en las escuelas de comunidades indígenas, donde principalmente predomina su lengua materna y cuyo contexto cultural es distinto al de las comunidades infantiles urbanas [...]

\footnotetext{
${ }^{3}$ Son diversos los casos que el Consejo en conjunto con la Comisión Nacional de Derechos Humanos (CNDH) ha atendido en relación con actos de discriminación a personas indígenas, entre ellos, en sectores como salud (negación a la atención médica o al servicio hospitalario), a la educación (negar la inscripción o ejercer violencia contra niños indígenas), entre otros. Señala el Consejo respecto a la discriminación por origen étnico que: "Es importante comprender que la discriminación rebasa el esfuerzo hecho en materia normativa y que es imprescindible que estos procesos sean acompañados de políticas integrales en favor de la inclusión y la igualdad de las personas" (Conapred, s.f.).
} 
El sistema educativo mexicano es discriminatorio de los pueblos indígenas en términos generales debido a los esquemas históricos de selectividad, la educación que se imparte no es pertinente, no está específicamente diseñada para atender sus necesidades de conocimiento, no se imparte en sus lenguas, no llega físicamente, o llega tarde y con pocos recursos, hasta las localidades que habitan. Asimismo, las condiciones socioeconómicas de marginación en las que viven las comunidades indígenas de nuestro país las limitan en muchas de sus oportunidades de desarrollo integral. Finalmente, los efectos lingüísticos que tiene sobre el aprendizaje el hecho de tener una lengua materna diferente del español, que es la lengua de enseñanza en México, son poco conocidos y peor atendidos desde la pedagogía escolar [...]

La discriminación perpetrada, causa un agravio a la población infantil indígena e impide el ejercicio pleno de sus demás derechos, tales como el derecho a la igualdad y a la educación de calidad. Asimismo, deja de observarse el principio del interés superior de las niñas y niños al no haber una plena satisfacción del ejercicio de su derecho a la educación reflejada en los resultados de instrumentos educativos estandarizados, que al aplicarse en poblaciones indígenas, pueden carecer de objetividad ya que no se adecuan a los contextos culturales, entorno social y de lenguaje predominante en ellos; esta situación afecta el derecho universal a la igualdad real de trato y de oportunidades de las personas, propiciándose por ello un acto de discriminación. (Conapred, 2011, pp. 26, 38-39)

Asimismo, como se mencionó anteriormente, durante muchos años la política castellanizante buscó la eliminación de las lenguas indígenas en las aulas, sancionando incluso a quienes se "atrevían" a hablar en su lengua materna. Se recomendaba a la vez a los padres de familia que no enseñaran a sus hijos y procuraran hablar en castellano frente a ellos. Esto provocó la desvalorización de la lengua así como su pérdida, pues los hablantes de la misma poco a poco se fueron reduciendo. Este tipo de concepciones conllevan un mensaje que induce al abandono de los elementos culturales a fin de lograr la inclusión en el sistema educativo, pero también en el social y laboral.

En resumen, como se ha argumentado hasta el momento, las políticas y acciones educativas enfocadas a la población indígena han estado enfocadas a la conversión, la dominación y la aculturación (Aguirre, 1975). Ello a partir de una inclusión - más en el sentido de la asimilación -, sin una verdadera consideración de sus elementos propios, es decir, el énfasis ha estado en el incremento en la cubertura y la matrícula escolar de las comunidades indígenas, y su traducción se ha dado en aspectos cuantitativos y no 
cualitativos. Asimismo, se ha insistido en la necesidad de omitir o invisibilizar sus elementos culturales, toda vez que resultan poco congruentes con los aspectos de la modernidad y la globalización, lo cual se ha traducido incluso en opiniones que ven como necesario que los indígenas dejen de serlo, para que el progreso nacional sea posible.

Al respecto, menciona Del Val (1993, p. 247) que "sus conocimientos, sus tradiciones, sus formas de relación social, [se han identificado] erróneamente como fuentes de la miseria y del atraso de la sociedad, y su erradicación se considera necesaria y saludable para el país". En la misma línea, Bartolomé (1996) señala que en la práctica, se busca desindianizar a "los indios" y es a través de los discursos y las acciones que continúan reproduciendo las ideas que históricamente han guiado la educación de las poblaciones indígenas, buscando así la homogeneización de la diversidad, la cual continúa asumiéndose como motivo de la desigualdad.

\section{Panorama actual de las escuelas indígenas en México 4}

En México, de manera oficial la educación indígena está destinada a los niveles de prescolar (de 3 a 5 años aproximadamente) y primaria (de 6 a 11 años aproximadamente $)^{5}$. Estadísticamente, representa alrededor del $10 \%$ de la población del sistema educativo nacional (Tabla 1) y se ubican generalmente en lugares donde las condiciones resultan complejas, pues se caracterizan por ser distantes, ubicarse en zonas de marginación o pobreza, donde suele haber carencia de servicios básicos o de salud, e incluso fuentes de trabajo. A su vez, estas dificultades se trasladan a los espacios educativos, donde se suman además cuestiones relacionadas con la organización escolar, la propuesta y materiales didácticas, así como las prácticas educativas.

\footnotetext{
${ }^{4}$ Salvo los casos donde se cita la fuente, la descripción que se presenta ha sido resultado de observaciones y entrevistas informales realizadas a partir de la visita a escuelas y docentes indígenas en diferentes estados de la República como Puebla, Veracruz, Oaxaca y la zona rural de la Ciudad de México.

${ }^{5}$ Sin embargo, es importante mencionar que además de estos dos niveles, existen otros servicios educativos que se imparten en algunas comunidades indígenas como la Educación inicial, la primaria comunitaria a cargo del Consejo Nacional para el Fomento Educativo (Conafe), la secundaria comunitaria, la telesecundaria, y para Educación Media Superior existen modalidades estatales de bachillerato indígena o intercultural. No obstante, de manera oficial, la instancia dedicada a nivel nacional de la educación indígena, la Dirección General de Educación Indígena (DGEI) trabaja sólo con los niveles mencionados inicialmente.
} 
Tabla 1. Alumnos, docentes y escuelas o planteles de sostenimiento público por nivel educativo para la Educación indígena en el ciclo escolar 2014-2015.

\begin{tabular}{|c|c|c|c|r|r|r|r|}
\hline $\begin{array}{c}\text { Tipo de } \\
\text { servicio }\end{array}$ & $\begin{array}{c}\text { Nivel } \\
\text { educativo }\end{array}$ & \multicolumn{2}{|c|}{ Alumnos } & \multicolumn{2}{c|}{ Docentes } & \multicolumn{2}{c|}{$\begin{array}{c}\text { Escuelas, } \\
\text { planteles }\end{array}$} \\
\hline \multirow{2}{*}{ Indígena } & Prescolar & 411,119 & $10 \%$ & 18,597 & $10 \%$ & 9,671 & $12.7 \%$ \\
\cline { 2 - 8 } & Primaria & 827,493 & $6.3 \%$ & 36,801 & $7.1 \%$ & 10,131 & $11.3 \%$ \\
\hline
\end{tabular}

Fuente: Elaborada con base en INEE (2016).

En cuanto a la infraestructura de estas escuelas, no obstante de que en la actualidad en el discurso y la política educativa se ha enfatizado en el apoyo material y económico, la realidad es que aún hay importantes carencias. Existen deficiencias materiales o recursos que no son de la suficiente calidad para atender a las poblaciones, dejando nuevamente a la educación de estas zonas en un grado de "tercera" o inferior a la que tienen las zonas urbanas o económicamente productivas. De acuerdo con los datos proporcionados por el Instituto Nacional para la Evaluación de la Educación (INEE, 2016, p. 58) un importante porcentaje de escuelas indígenas tienen el techo de lámina de asbesto, metal, cartón, palma o carrizo, o éste es inexistente; en algunos centros educativos las paredes son de tablarroca, lámina, madera, o incluso no las tienen; los pisos son de tierra o materiales movibles. En cuanto a servicios básicos:

Más de la mitad no tiene agua todos los días de la semana, y entre una tercera parte y casi la mitad carecen de energía eléctrica. Estas condiciones se explican en gran parte por las características de infraestructura de las localidades, pues ese tipo de escuelas suelen estar ubicadas en comunidades rurales con altos o muy altos grados de marginación, que en ocasiones no disponen de servicios públicos. (INEE, 2016, pp. 58 y 60)

En lo concerniente a condiciones básicas relacionadas con la salud e higiene, no todas las escuelas tienen excusados, o disponibilidad de agua para lavarse las manos o beber (bebederos). En cuanto a medidas de seguridad, hay escuelas que presentan vidrios rotos o estrellados, techos en mal estado, instalaciones eléctricas deficientes (cuando las hay) y paredes agrietadas. 
En relación con la organización escolar, debido a que muchas veces estas escuelas están ubicadas en lugares de difícil acceso y donde la matrícula escolar es pequeña, se considera innecesario asignar a más de un docente, por lo que faltan escuelas con plantillas docentes completas, lo que ha implicado la instalación de escuelas multigrado. En éstas, la cantidad de docentes es distinta al número de grados, por ejemplo, un docente puede atender dos o más grupos, en vez de uno como ocurre en la escuela regula; existen incluso algunas donde uno solo atiende los tres grados en preescolar o los seis grados de primaria.

Estas escuelas surgen en México ${ }^{6}$ debido a "la necesidad de atender a los niños y niñas que viven en comunidades pequeñas y aisladas a lo largo del país, casi todas en zonas rurales y de las cuales una gran parte se ubica en comunidades indígenas" (Gallardo, 2004, p. 1). Según datos del INEE (2016), actualmente dos de cada tres escuelas indígenas son de este tipo.

De acuerdo con Weiss (2007) que entre los problemas a los que se enfrentan las escuelas multigrado en la actualidad están:

\footnotetext{
${ }^{6}$ La historia de la escuela multigrado en México tiene antecedentes remotos, pues durante mucho tiempo las escuelas funcionaron sin una organización en grados o basada en las edades. Fue hasta el siglo XIX que la escuela se fue graduando y se comenzaron a establecer de forma inicial programas de estudios y momentos de evaluaciones. Hacia el siglo XX, la organización graduada comenzó a establecerse como normal, sin considerar que algunas escuelas no tenían la posibilidad para organizarse así, lo que obligo en algunos casos al cierre de escuelas con un solo maestro y que los niños se desplazaran a lugares remotos para recibir educación. En los años posteriores a la Revolución Mexicana (1910-1921 aproximadamente), el discurso en torno a la escuela de organización graduado continuo, prestando poca atención a aquellas que contaban con un solo docente. En algunos casos, para atender a los niños indígenas se fundaron internados alejados de las comunidades de origen, y se consideraba que de este modo era más fácil que abandonaran sus costumbres. Entre 1940 y 1970, diversas acciones apuntaban a la atención de las escuelas rurales: el Primer Congreso Nacional de Educación Rural, la creación de Instituto Federal de Capacitación del Magisterio (IFCM), la publicación y circulación de libros acerca de la escuela rural unitaria, la fundación del Centro Regional de Educación Fundamental de América Latina (CREFAL), las recomendaciones de la UNESCO en relación a las escuelas indígenas. Dadas las dificultades para lograr acuerdos entre el sindicato de maestros y la Secretaría de Educación Pública para establecer escuelas en comunidades alejadas, la SEP creo en 1971 el Consejo Nacional de Fomento Educativo (Conafe) con la intención de atender a las primarias de esas zonas y se diseñó un modelo pedagógico específico. A la par, la Dirección General de Educación Indígena continuo haciéndose cargo de algunas zonas donde habitaban principalmente comunidades indígenas con un sistema similar al de internados. En el ciclo escolar 1991-1992 se puso en marcha el Programa para abatir el rezago educativo (PARE) el cual buscaba atender los problemas de rezago educativo, principalmente en el medio rural e indígena, y apoyando a las escuelas multigrado en relación a la capacitación, actualización, materiales educativos, equipamiento, infraestructura, entre otros (Rockwell y Garay, 2007, pp. 9-32).
} 
- Rotación constante de docentes durante el ciclo escolar, dejando a los grupos sin maestro hasta la asignación de otros, lo que incluso puede tardar meses.

- Desempeño de labores de dirección, además de las docentes, lo cual implica la suspensión de labores cuando se requieren hacer trámites en la supervisión.

- Dado el horario de jornada escolar, resulta casi imposible la atención por grado; además de que se debe prestar mayor atención al primer grado, sobre todo en relación al proceso de adquisición de escritura.

- Cuando la atención se da por grados, mientras el docente asigna tareas a un grupo, los otros no tienen atención. En algunos casos, para mantenerlos "ocupados" se emplean actividades como el copiado que son poco congruentes con los enfoques de enseñanza.

Retomando a las escuelas indígenas, además de la diversidad étnica, cultural y lingüística $^{7}$ - que caracterizan a las comunidades indígenas - los docentes se enfrentan al reto de desempeñar diversas acciones no sólo como profesor, sino como director, gestor, administrador, etcétera, ante la falta de las figuras respectivas para desempeñar dichas acciones.

A pesar que desde los planteamientos de la interculturalidad, la diversidad puede significar una valiosa oportunidad para el desarrollo de los procesos de aprendizaje, la realidad es que, debido a las diferentes carencias, la escuela multigrado representa desafíos importantes. Significa, primero, atender a alumnos de diferentes grados en un mismo salón, asignando tareas en intervalos de tiempo para cada uno, o por otro lado, usar un sistema globalizador en el que el grado de explicación sea diferente a cada grado, pero que deje a veces de lado, la profundidad con que se pueden atender los temas. En consideración de Rosas (2009), en las escuelas multigrado:

\footnotetext{
${ }^{7}$ Existen escuelas indígenas donde asisten alumnos pertenecientes a diversos grupos étnicos, por lo que en algunas ocasiones es posible hablar de aula bilingües e incluso trilingües. En reciente años, los procesos migratorios ha favoreciendo esta diversidad en las aulas; en otros casos se debe a la ubicación de las propias escuelas en medio de comunidades de afluencia de varios grupos indígenas.
} 
Se vive y se trabaja en una continua contradicción: tanto el currículum como los materiales educativos están diseñados para las escuelas llamadas completas o regulares, que cuentan con un maestro para cada grado, ignorando la situación de las escuelas multigrado. Los resultados de aprendizaje son bajos, debido a que sus maestros no están preparados, ni cuentan con la asesoría los materiales y la infraestructura adecuados. (p. 2)

Respecto a los materiales tradicionalmente usados, los libros de texto por ejemplo, están destinados a cada grupo sin considerar que se encuentran bajo una modalidad en la que no es posible atender con suficiente profundidad los temas que se contemplan para una escuela regular.

Asimismo, si bien es cierto que se ha declarado la importancia de preservar las lenguas indígenas, lo cierto es que los materiales didácticos y los libros de texto siguen siendo en español. A la vez, no se ha logrado consolidar una estrategia que implique el uso de la lengua no sólo en el espacio destinado curricularmente para ello, sino para el trabajo en otras asignaturas como matemáticas o ciencias, lo que obliga a abandonar la lengua materna y no llevar su desarrollo durante toda la educación básica.

En este mismo desarrollo de habilidades comunicativas, los alumnos se enfrentan al aprendizaje de una segunda lengua (el español), lo que genera un proceso distinto al que se destina a las escuelas no indígenas, quienes prestarán una mayor atención a un solo idioma y no dos. Esto implica además que la brecha entre escuelas sea más grande, pues no se busca que los alumnos de escuelas regulares se acerquen a los conocimientos indígenas ni a su lengua.

En cuanto al uso de las TIC, además del reto de la poca disponibilidad que hay de ellas en las escuelas indígenas, hay otros retos tanto para los profesores como para los alumnos. Los primeros requieren de actualización para poder usarlas e implementarlas en su práctica educativa, y por el lado de los alumnos, la posibilidad únicamente de acceso al internet y la computadora en el medio escolar, sin posibilidad de ensayar o practicar en casa.

Retomando lo relativo a las lenguas indígenas, existen dificultades adicionales a las ya mencionadas. Aunque de manera deseable se establece que los docentes que impartan educación en estas escuelas deben hablar la 
lengua de la comunidad, la realidad es que no sucede así. Muchos docentes son enviados a lugares donde la lengua no es la misma que dominan, debido a cuestiones administrativas y sindicales $^{8}$ que no consideran este aspecto. A la vez, se han empleado a algunos que no tienen conocimiento de ninguna lengua indígena ni tampoco un acercamiento a estas poblaciones, generándose así procesos educativos poco pertinentes con la visión y organización que tienen las comunidades.

En relación al planteamiento pedagógico, generalmente, al tratarse el tema de la diversidad étnica e indígena del país se cae en estereotipos como asociarla con el México prehispánico o la admiración hacia las artesanías y el folclor. De esta forma, el indígena se presenta como un sujeto pasivo de la historia (Dosil y Lepe, 2011 , p. 5), que carece de voz propia y sin aportaciones significativas a la identidad y patrimonio nacional, más allá de algunas danzas o "platillos típicos". Asimismo, en los materiales y la narrativa de distintos contenidos se invisibiliza la participación de las comunidades indígenas, no sólo en lo que respecta a los distintos momentos de la historia nacional, sino en diferentes aspectos como el cuidado del ambiente o la organización política y social. Ante la falta de una verdadera inclusión de la diversidad étnica del país, las actividades en las aulas suelen centrarse en aspectos triviales relacionadas con el folclor, las costumbres y tradiciones, pero sin una verdadera reflexión y comprensión, tanto por parte de estudiantes como de profesores.

Un aspecto a resaltar es que si bien pudiera pensarse que en las comunidades indígenas existe una valoración por la cultura y sus componentes, la tensión que existe en la actualidad entre lo global y lo local ha provocado cambios en las concepciones de las personas, encontrándose así la aspiración e imitación de modelos de vida más occidentales, y el no querer aprender la lengua indígena por considerarla obsoleta, carente de valor o que provoca el cierre de oportunidades, prefiriéndose así que se enseñe en la

\footnotetext{
${ }^{8}$ Hasta antes de la regulación del Sistema Profesional Docente por parte del gobierno mexicano, existían diversas prácticas en relación con la asignación de plazas docentes. En algunos casos, docentes de recién ingreso eran enviados a comunidades indígenas - incluso sin pertenecer a un grupo originario o hablar alguna lengua - en tanto lograban su cambio a una escuela más cercana a los centros urbanos. En otros casos, algunos docentes eran enviados a escuelas remotas "como castigo" ante la falta de una norma o por haber tenido problemas en el lugar donde laboraban, para "alejarlos" del conflicto.
} 
escuelas el inglés, toda vez que dentro de algunas comunidades los procesos migratorios han aumentado considerablemente y forman parte del proyecto de vida de algunos estudiantes. Esta realidad demuestra que el Estado tiene deficiencias y deudas con las escuelas indígenas.

\section{La educación indígena vista desde la inclusividad e interculturalidad}

Como se ha descrito anteriormente, la atención educativa a las comunidades indígenas ha sido un proceso más o menos lento, y en ocasiones con una perspectiva alejada de las necesidades de los grupos originarios, quienes históricamente continúan estando en desventaja y marginación por parte del sistema educativo. Los retos presentes en las escuelas indígenas suponen una serie de esfuerzos encaminados no sólo a cuestiones de política y administración educativa o la generación de espacios físicos, sino en lo referente a la formación inicial y continua de los profesores, la generación de materiales, la participación de la sociedad, y un planteamiento pedagógico y didáctico pertinente que favorezca los procesos de aprendizaje desde las perspectivas de la inclusividad y la interculturalidad.

Son muchos los desafíos por afrontar pues la realidad de las escuelas, su funcionamiento, prácticas y cultura dista aún de lo básico y deseable. La atención o desatención de estas esferas puede generar condiciones para el cambio hacia la inclusión, o por el contrario, reforzar el estancamiento de la escuela.

En México, en materia educativa se disponen de diferentes políticas encaminadas a mejorar las condiciones de las escuelas y lograr la inclusión de niñas, niños y jóvenes en edad escolar, por lo que las aulas representan espacios heterogéneos no sólo en lo que refiere a la atención a necesidades educativas especiales, sino considerando estilos de aprendizaje, niveles socioeconómicos, diversidad lingüística y étnica, etcétera.

Sin embargo, la inserción de alumnos en el sistema no ha traído consigo necesariamente un cambio en aspectos como la organización curricular, la generación de materiales, las prácticas educativas, entre otros, generando así mecanismos de exclusión, sobre todo hacia aquellos alumnos que se 
encuentran en situaciones de vulnerabilidad, presentándose así dificultades para desarrollar sus procesos de aprendizaje en las escuelas tal como funcionan hasta ahora.

Una verdadera escuela con carácter inclusivo considera a la diversidad como una oportunidad valiosa para el desarrollo de los procesos de enseñanza y aprendizaje y los organiza con base esta heterogeneidad. Según Fernández (2003, como se citó en Valenciano, 2009):

[Las escuelas inclusivas] deben reunir una serie de condiciones hacia las cuales es necesario avanzar progresivamente considerando este principio como un elemento que enriquece el desarrollo personal y social, como un proyecto educativo de toda la escuela que pretende implementar un currículo susceptible de ser adaptado a las diferentes capacidades, motivaciones, ritmos y estilos de aprendizaje de los alumnos. (p. 20)

En cuanto a la parte intercultural, existen también retos importantes a superar para lograr que los estudiantes, independientemente de sus condiciones u origen étnico, alcancen el máximo desarrollo de sus facultades a partir de los procesos formativos y poder así continuar en su formación en los diferentes niveles, o bien, poder desempeñarse como ciudadanos en sus espacios de convivencia y de acuerdo con sus posibilidades de participación. Ello significa la posibilidad de un replanteamiento que parta de las necesidades y características de la propia comunidad, es decir, una educación que pertenezca al contexto.

Este planteamiento contraviene la postura etnocéntrica, que parte de la creencia de que existe una cultura dominante y superior, ante la cual, las que no corresponden con ella, son inferiores o poseen un valor menor. De este modo, se concibe a la diversidad como una dificultad que limita o imposibilita el trabajo y ante lo cual hay que emprender acciones de homogeneización, principalmente en lo que respecta al idioma, para poder llevar a cabo los procesos en el aula. Esto se debe a que:

El bilingüismo pedagógico todavía se define sobre todo como un "bilingüismo sustractivo" (en el que una lengua se enseña, paradójicamente, para hacerla desaparecer) y no como "bilingüismo aditivo" (en el que una lengua se enseña para que pueda usarse además de otra u otras). (De la Peña, 2002, pp. 49-50) 
Además, esta postura etnocéntrica justifica la exclusión, posicionando a la discriminación como un acto social legitimo a favor del progreso y desarrollo, avalando la subordinación hacia quienes no cumplen con los parámetros y estándares sociales. Las connotaciones negativas buscan enmarcar características considerando a los indígenas como ignorantes, atrasados y carentes de cultura. De este modo, existe un marco cultural detrás de los actos de discriminación y violencia hacia los indígenas a partir de dichos estereotipos.

En contraste, mirar el currículo escolar desde la interculturalidad implica superar el enfoque eurocéntrico para explicar la historia, la revisión de la geografía a partir del contexto más cercano, el acercamiento a las narraciones indígenas o a las expresiones artísticas de los pueblos originarios, entre otros ejemplos (Jiménez, 2012, p. 15). En cada uno de ellos, la socialización y el intercambio de experiencias juega un papel importante para los procesos de enseñanza y aprendizaje, no sólo en virtud de los contenidos, sino de las habilidades y valores que se pueden desarrollar en conjunto.

No obstante, girar el rumbo hacia el contacto cultural resulta insuficiente cuando se queda ahí. Si bien es cierto que ha sido un gran acierto y avance el reconocimiento del pluralismo en las aulas, y que se ha logrado que en un mismo espacio converjan distintas culturas, es importante que se den pasos más allá. Desde una mirada multicultural se hace el reconocimiento del otro, pero no se pretende una atención, sino bajo una visión de diferencia. Ésta se entiende como:

El reconocimiento del otro como distinto, pero no implica necesariamente el establecimiento de relaciones igualitarias entre los grupos, la historia muestra como se ha exigido a "los otros" desaparecer en tanto grupo cultural, ya sea por medio del etnocidio directo como por sus derivaciones menos violentas, aunque constituidas en torno al mismo objetivo; destaca aquí la asimilación y la integración como políticas adoptadas por los estados nacionales frente a sus pueblos originarios. (Gallardo, 2004, p. 4)

En contraste, la interculturalidad propone el establecimiento de espacios en el que todos encuentren cabida, pero que además, sea posible compartir y generar conocimientos, ideas y pensamientos que conjuguen la participación 
de cada uno de los integrantes. Este avance se vincula además con la relación entre los integrantes de la comunidad escolar reconociéndose como sujetos que posee conocimientos y saberes que han configurado a través de su vida cotidiana y que forman parte de su cultura y formas de ver y explicarse el mundo.

En este intercambio, existen propuestas que invitan a favorecer una educación bilingüe en todas las escuelas, no sólo las indígenas, permitiendo así el combate a aspectos como la discriminación y el racismo (Hamel, 2001, como se citó en Schmelkes, 2001). En el mismo sentido, Latapí (2001) menciona que:

Mucho pueden hacer los profesores para que los alumnos comprendan y aprecien rasgos valiosos de la organización social que subsiste hasta nuestros días: su sentido comunitario, expresado en los servicios gratuitos a la comunidad (cargos, mayordomías, tequios, faenas, etc.), el respeto a los ancianos o la valoración de la tierra que puede fundamentar maravillosamente la urgente cultura ecológica; que aprecien el sentido del símbolo y de la ceremonia, la importancia ritual del obsequio, y ponderen otros elementos de la cosmovisión indígena (p.7).

Pensar en las escuelas multigrado representa una oportunidad muy valiosa si su implementación se lleva a cabo en condiciones más adecuadas, no sólo dentro de los contextos indígenas, sino incluso en los espacios urbanos y las escuelas regulares. Una de sus bondades más importantes de este tipo de organización radica en la posibilidad de que niños de diferentes edades y diferentes grados escolares puedan compartir sus referentes a través de diversas prácticas donde las concepciones y experiencias de cada uno se vean inmersas en los procesos. Asimismo, se puede reflejar en el apoyo común y asesoramiento conjunto, tanto de docentes como alumnos, para el logro de los aprendizajes, y a la vez, de valores y actitudes encaminadas a favorecer la convivencia pacífica y respetuosa de la diversidad. 


\section{Comentarios finales}

En este trabajo se ha querido presentar la orientación que ha tenido la educación indígena en México, así como las dificultades que presentan las escuelas de este tipo y que dificultan sus procesos de enseñanza y aprendizaje.

El discurso en torno a la educación en comunidades indígenas, nos da cuenta de una serie de problemáticas a nivel didáctico, tecnológico, de administración, gestión, entre otros. La transformación educativa en estos sectores, requiere de enfrentarse a estas grandes dificultades, pues no es posible negar, a pesar de las intencionalidades utópicas que se tengan, que es necesario atender a ciertas cuestiones básicas que redunden en beneficios para los procesos educativos que se llevan a cabo en las escuelas.

Como parte de su labor, la política educativa deberá retomar su compromiso social hacia las escuelas indígenas para coadyuvar el desarrollo armónico e integral de niñas, niños y jóvenes indígenas eliminando sesgos institucionales que de manera velada ha adoptado y continúa reproduciendo a través de sus diferentes acciones. Ello implica que la educación indígena favorezca la problematización acerca de la realidad y los problemas que históricamente han enfrentado asumiéndose como sujetos activos para participar en la medida de sus posibilidades.

En tanto, como parte de las demás escuelas, tanto urbanas como rurales no indígenas, se debe coadyuvar a la construcción de una ciudadanía que reconozca a la diversidad como un valor importante y no como una traba, y que a la vez valore la relevancia de la cohesión social de todas las personas; es por tanto necesario, replantear el trabajo escolar con experiencias educativas significativas que permitan la erradicación de problemas como el racismo, la discriminación, la xenofobia y la violencia.

Repensar la educación desde una visión intercultural e inclusiva implica el reconocimiento y revisión histórica en torno a las desigualdades que han existido y que se encuentran vigentes hasta nuestros días. A través del diálogo y la participación, se deberá buscar el reconocimiento y la valoración de la 
diversidad cultural, a través de una praxis educativa que se traslade a otras esferas sociales a partir de valores como el respeto, equidad y justicia social.

Se requiere dejar de ver desde arriba y desde el centro para poder emprender una labor en la construcción conjunta. Por ello, en medio de las dificultades que en el presente existe, es importante resaltar el esfuerzo que realizan las comunidades para el mejoramiento de sus escuelas, a través de la organización entre sus habitantes y la gestión ante diferentes niveles de gobierno con el fin de obtener mayores recursos.

\section{Referencias Bibliográficas}

Aguirre, G. (1975). Teoría y práctica de la educación indígena. México: SEP. Antolínez, I. (2013). La gestión de la diversidad cultural en España y México: construcción de la diferencia y exclusión en educación intercultural. Revista Latinoamericana de Educación Inclusiva, 7 (2), 115-132.

Bartolomé, M. (1996). Pluralismo cultural y redefinición del Estado en México. Brasilia: Departamento de Antropología, Universidad de Brasilia.

Consejo Nacional de Evaluación de la Política de Desarrollo Social (Coneval). (2012). La pobreza en la población indígena de México. Recuperado de http://www.coneval.org.mx/Informes/Coordinacion/INFORMES_Y_PUBLICACI

ONES_PDF/POBREZA_POBLACION_INDIGENA_2012.pdf

Consejo Nacional para Prevenir la Discriminación (Conapred), (s.f.). Discriminación en contra de la población indígena en México. Recuperado de http://www.conapred.org.mx/index.php?contenido=noticias\&id=798\&id_opcion= $303 \&$ op $=448$

Consejo Nacional para Prevenir la Discriminación (Conapred). (2010). Encuesta Nacional sobre Discriminación en México, ENADIS 2010. Resultados sobre diversidad cultural. México: Conapred.

Consejo Nacional para Prevenir la Discriminación (Conapred). (2011, 22 de febrero). Resolución por disposición: 1/2011. Recuperado de http://www.conapred.org.mx/userfiles/files/RPD_01_2011_Accs_VP(2).pdf

Constitución Política de los Estados Unidos Mexicanos. Artículo $2^{\circ}$. Recuperado de http://www.diputados.gob.mx/LeyesBiblio/pdf/1_240217.pdf

De la Peña, G. (2002). La educación indígena, consideraciones críticas. Sinéctica, (20): 46-53.

Del Val, J. (1993). El indigenismo. En L. Arizpe (Coord.), Antropología breve de México. México: Academia de la Investigación Científica. CRIM. UNAM.

Dosil, F. y Lepe, L. (2011). La educación ante el nudo de significados de la interculturalidad. En XI Congreso Nacional de Investigación Educativa (pp. 1-8). México: Consejo Mexicano de Investigación Educativa. 
Fernández, A. (2003). Educación Inclusiva: Enseñar y aprender entre la diversidad. Revista Digital Umbral 2000. Recuperado de http//www.portal.perueduca.edu.pe/basicaespecial/artculos/art01_01-09-06.pdf Galeana, R. (2013). Ser niña, niño o adolescente indígena en México y los obstáculos para ejercer el derecho a la educación. Rayuela, (9): 89-97.

Gallardo, A. (2004). Las escuelas multigrado frente a la diversidad cultural, étnica y lingüística. Horizontes de posibilidad desde la educación intercultural. En Reunión Nacional Propuesta Educativa Multigrado. Fase extensiva. Mejorar la enseñanza y el aprendizaje. México.

Gamio, M. (1916). Forjando Patria (Pro nacionalismo). México: Porrúa.

Gutiérrez, E. (2006). Baja cobertura y calidad de la educación indígena. Caracas: Fundación Escuela de Gerencia Social y Ministerio del Poder Popular para la Planificación y Desarrollo.

Hecht, A. (2007). Educación intercultural bilingüe: de las políticas homogeneizadoras a las políticas focalizadas en la educación indígena argentina. Revista Interamericana de Educación de Adultos, 29, 65-85.

Hernández, L. (2013, 25 de agosto). Prueba Enlace: simulación y discriminación. La Jornada. Sección: Opinión. Recuperada de http://www.jornada.unam.mx/2013/06/04/opinion/017a2pol

Instituto Nacional de Estadística y Geografía (Inegi). (2011). Población de 3 años y más en entidades federativas seleccionadas y su distribución porcentual según condición de autoadscripción étnica para cada entidad federativa, sexo y condición de habla indígena. Censo de Población y Vivienda 2010. Recuperado de

http://www3.inegi.org.mx/sistemas/TabuladosBasicos/LeerArchivo.aspx?ct=274 $95 \& \mathrm{c}=27303 \& \mathrm{~s}=\mathrm{est} \& \mathrm{f}=2$

Instituto Nacional de Lenguas Indígenas (Inali). (2008). Programa de Revitalización, Fortalecimiento y Desarrollo de las Lenguas Indígenas Nacionales 2008-2012. México: Inali.

Instituto Nacional para la Evaluación Educativa (INEE). (2016). La educación obligatoria. México: INEE.

Jiménez, F. (2012). Violencia escolar en contextos educativos multiculturales: Una aproximación desde los modelos de gestión de la diversidad cultural. Psicoperspectivas, Individuo y Sociedad, 11(2): 8-30.

Latapí, P. (2001, 18 de agosto). Multiculturalidad: ¿qué es eso? Revista Proceso. Recuperado de http://www.proceso.com.mx/186244/multiculturalidadque-es-eso

Martínez, E. (2011). La educación indígena e intercultural en México y sus implicaciones en la construcción de ciudadanías. En XI Congreso Nacional de Investigación Educativa (pp. 1-9). México: Consejo Mexicano de Investigación Educativa. 